\title{
Imagerie et fertilité, quelles conséquences pour l'andrologue ? Imagerie du scrotum vue par le radiologue
}

\author{
Imaging and fertility, what consequences for the andrologist? \\ View scrotal imaging by the radiologist
}

\section{T. Puttemans}

Reçu le 10 septembre 2009 ; accepté le 14 octobre 2009

(C) SALF et Springer-Verlag France 2009

\begin{abstract}
Résumé L'échographie scrotale fournit à l'andrologue des informations iconographiques, susceptibles de changer fondamentalement la prise en charge du patient hypofertile. La recherche de signes d'obstruction canalaire, la détection de pathologies testiculaires infracliniques et l'évaluation de la perfusion testiculaire sont les véritables challenges pour le radiologue. L'échographie du contenu scrotal doit être proposée à tout patient qui consulte pour infertilité, au même titre que le bilan biologique et spermiologique, car elle oriente le diagnostic clinique, permet la détection de pathologie occulte et influence la stratégie thérapeutique.
\end{abstract}

\section{Mots clés Infertilité $\cdot$ Échographie scrotale $\cdot$ Radiologue}

\begin{abstract}
Scrotal ultrasound provides andrologist iconographic informations likely to fundamentally change the management of subfertile patients. Looking for tubular obstructive signs, the detection of infraclinic testicular pathologies and the testicular perfusion evaluation are the real challenges for the radiologist. Ultrasonography of the scrotal contents should be offered to all patients who consulted for infertility, as well as biological and spermiological assessment, because it directs clinical diagnosis, allows the detection of occult disease and influence therapeutic strategy.
\end{abstract}

Keywords Infertility $\cdot$ Scrotal ultrasonography ·

Radiologist

\section{Introduction}

L'échographie du système urogénital est recommandée chez l'homme hypofertile, particulièrement en cas en cas

T. Puttemans $(\bowtie)$

Imagerie médicale, clinique Saint-Pierre, avenue Reine-Fabiola,

9, B-1340 Ottignies-Louvain-la-Neuve, Belgique

e-mail : thierry.puttemans@scarlet.be d'examen clinique difficile ou de terrain favorable au développement d'une pathologie tumorale testiculaire [1-5].

Cet examen comporte l'exploration, par voie externe, du testicule et du tube excréteur, jusqu'au pli inguinal, et l'exploration, par voie endorectale, du carrefour urogénital.

Il apporte à l'andrologue des éléments iconographiques d'orientation diagnostique, en faveur d'une origine sécrétoire ou excrétoire à l'infertilité.

L'imagerie du contenu scrotal, détaillée dans cette revue, est, au même titre que l'imagerie du carrefour urogénital et des reins, une des étapes incontournables de la mise au point. La détection de pathologies testiculaires infracliniques, fréquemment associées à l'infertilité, comme la tumeur testiculaire, est le véritable challenge pour le radiologue, car susceptible de modifier fondamentalement la prise en charge clinique du patient hypofertile. L'évaluation de la vascularisation testiculaire grâce à l'utilisation du Doppler couleur, de l'analyse spectrale et, apparue plus récemment, de l'échographie de contraste, offre de nouvelles perspectives intéressantes pour l'évaluation du potentiel testiculaire de la spermatogenèse et pour la guidance du prélèvement de spermatozoïdes.

\section{Techniques d'exploration}

L'échographie du contenu scrotal, réalisée à l'aide d'une sonde linéaire de haute fréquence, comporte l'étude précise de la topographie, du volume et de la texture du testicule, de la position, de la taille et de l'aspect de l'épididyme et du canal déférent [6].

L'échographie-Doppler testiculaire comporte une analyse couleur de la densité vasculaire et une étude spectrale du signal artériel intratesticulaire [7].

\section{Aspects normaux et variantes courantes}

Le testicule normal a une forme ovale, une échogénicité élevée, une échostructure homogène et des contours réguliers. 
Le hile testiculaire se reconnaît à sa forme triangulaire et son hyperéchogénicité. La vascularisation testiculaire est homogène avec disposition radiaire des artères intratesticulaires et périphériques de l'artère capsulaire et ses branches.

Une des variantes les plus fréquentes est l'existence d'une artère transtesticulaire qui apparaît sous la forme d'une ligne hypoéchogène, intratesticulaire.

L'épididyme et le déférent sont explorés depuis la tête épididymaire jusqu'au segment funiculaire du canal déférent [8].

La tête épididymaire, de forme triangulaire et d'échogénicité comparable à celle du testicule, est observée, dans $97 \%$ des cas, au-dessus du pôle supérieur du testicule. Dans $3 \%$ des cas, elle est localisée sous le pôle inférieur du testicule et est associée à une rotation complète de l'épididyme. Le corps et la queue épididymaires sont moins échogènes que la tête et sont généralement observés au versant antérolatéral du testicule. Dans $6 \%$ des cas, ils sont observés sous le testicule.

L'anse épididymodéférentielle présente un aspect typique en $U$, et le segment initial du canal déférent apparaît habituellement tire-bouchonné, à l'inverse du segment funiculaire du déférent qui apparaît rectiligne.

La paroi musculaire du canal déférent lui confère un aspect fortement hypoéchogène, et la lumière déférentielle est visible sous la forme d'un spot hyperéchogène central.

Le segment funiculaire du déférent peut être différencié aisément des vaisseaux funiculaires grâce au Doppler couleur.

\section{Anomalies fréquemment associées à l'infertilité}

\section{Anomalie de volume testiculaire}

Le volume testiculaire adulte, calculé automatiquement par l'appareil d'échographie (longueur $\times$ largeur $\times$ épaisseur $\times 0,71$ ) est normalement compris entre 15 et $30 \mathrm{ml}$ (moyenne : $26 \mathrm{ml}$ ). Ce calcul échographique est plus précis que l'examen clinique, particulièrement en présence d'une hydrocèle ou d'une ectopie, et donne systématiquement des valeurs plus faibles que le calcul du volume par l'orchidomètre de Prader (moyenne : $36 \mathrm{ml}$ ) [9].

Dans la population de sujets infertiles, le volume testiculaire moyen est plus faible ( 9 à $19 \mathrm{ml}$ ). Cela s'explique notamment par la fréquence élevée, dans cette population, de varicocèle et d'atrophie testiculaire secondaire (cryptorchidie, torsion, infection).

Cette atrophie testiculaire est particulièrement marquée en cas d'azoospermie sécrétoire, où $100 \%$ des testicules droit et gauche ont moins de $10 \mathrm{ml}$ (moyenne : 8,3 ml) [10].

Cette diminution du volume testiculaire chez le sujet infertile est corrélée avec la diminution du nombre de spermatozoïdes et la perte de fonction testiculaire $[9,11]$.

Cette perte de volume, surtout si elle est bilatérale, est un bon signe échographique en faveur d'une origine sécrétoire à l'azoospermie : dans la population de sujets azoospermiques excrétoires, le volume testiculaire est significativement plus élevé que dans la population de sujets azoospermiques sécrétoires [12].

\section{Anomalie de topographie testiculaire}

L'échographie est très performante pour localiser le testicule dans le cordon et en étudier le volume et la structure.

Cela est particulièrement vrai chez les patients obèses ou en cas d'antécédent de chirurgie inguinale pour hernie, où la palpation testiculaire peut être rendue difficile.

En cas de cryptorchidie, la résonance magnétique peut être utile pour localiser le testicule lorsque celui-ci est intra-abdominal [10].

\section{Anomalie d'échogénicité testiculaire}

Une hypoéchogénicité testiculaire globale est fréquemment observée chez le sujet hypofertile et est due à l'atrophie testiculaire (perte cellulaire, augmentation de la composante conjonctive intercellulaire et épaississement des septa interlobulaires).

Ainsi, le testicule cryptorchide (non descendu) apparaît classiquement plus hypoéchogène que le testicule normal : cette modification d'échogénicité est due à l'atrophie testiculaire, mais, aussi, aux conditions de recueil des images échographiques (absorption du faisceau d'ultrasons par les tissus cellulograisseux du cordon). Même après correction chirurgicale de la cryptorchidie, le testicule restera hypoéchogène.

Cette hypoéchogénicité rend plus difficile la détection d'une éventuelle lésion tumorale hypoéchogène, car celleci se confond avec le parenchyme testiculaire environnant. On notera qu'une hypoéchogénicité testiculaire globale, relative, peut aussi être observée, occasionnellement, chez le sujet normal, lorsque des enveloppes scrotales épaisses absorbent le faisceau d'ultrasons.

Une hypoéchogénicité testiculaire, en nappe, épousant grossièrement l'anatomie lobulaire du testicule, est souvent observée après un épisode ischémique ou infectieux et constitue la trace indélébile de l'agression parenchymateuse antérieure et de l'atrophie qui en résulte. Ces remaniements hypoéchogènes ne sont pas spécifiques de l'infertilité, mais y sont plus fréquemment retrouvés, le degré d'hétérogénicité testiculaire étant négativement corrélé avec la qualité du sperme [13].

Le nodule intratesticulaire a lui aussi, dans la majorité des cas, un aspect hypoéchogène. Cela en facilite la détection (sauf en cas de petit testicule hypoéchoène, cryptorchide). Histologiquement, il s'agira de tumeur des cellules de Leydig (31\%), de séminome (25\%), de tumeur nonséminomateuse $(14,5 \%)$, de tumeur des cellules de Sertoli 
(4,5\%), de tumeurs bénignes (adénomateuses, hyperplasie des cellules de Leydig) [25 \%] [14].

La prévalence du nodule intratesticulaire varie selon la cause de l'infertilité : faible en cas d'infertilité d'origine féminine, elle augmente fortement en cas d'antécédent de cryptorchidie traitée hormonalement ou chirurgicalement [15].

La fréquence des tumeurs testiculaires est plus élevée chez les patients infertiles $(1 / 200)$, considérés « à risque », que dans la population générale (1/20 000) [3].

Toutefois, pour Raman et al., le risque de trouver un vrai cancer non palpable par échographie reste faible dans une population de sujets infertiles $(0,3 \%)$, ce qui ne justifie pas un screening échographique systématique [16].

L'attitude pratique, actuellement préconisée, en cas de découverte d'un nodule non palpable, dépendra de la taille du nodule, de son aspect échographique et des données cliniques. Le nodule intratesticulaire est soit :

- unique, inférieur à $5 \mathrm{~mm}$, hypoéchogène, arrondi, bien limité, non vascularisé en mode Doppler couleur, non palpable, découvert fortuitement à l'occasion d'une échographie de dépistage. Ce type de nodule ou incidentalome est de plus en plus fréquemment découvert. En cas de marqueurs tumoraux négatifs, ce qui est généralement la règle, ces nodules, considérés comme bénins (tumeur de Leydig, hyperplasie des cellules de Leydig), sont surveillés par échographie simple et ne nécessitent pas d'exérèse d'emblée [17] ;

- unique, supérieur à $5 \mathrm{~mm}$, hypoéchogène, vascularisé en mode Doppler ou non, non palpable. Ce type de nodule nécessite une exploration biopsique systématique, pratiquée dans le même temps que le prélèvement de spermatozoïdes par aspiration, et une éventuelle exérèse à visée curative : en effet, le risque histologique de trouver un cancer augmente, particulièrement dans la souspopulation de patients azoospermiques présentant un syndrome de type " cellules de Sertoli seules » et en cas d'antécédent de cryptorchidie ou d'hypogonadisme gonadotrophique [18]. Un certain nombre de ces nodules enlevés ne seront pas des cancers (petite tumeur de Leydig, hyperplasie des cellules de Leydig), mais la biopsieexérèse est justifiée par l'incertitude quant au devenir des tumeurs de Leydig et par le fait que la biopsie testiculaire va révéler l'existence de carcinome in situ (CIS) $[18,19]$. Une alternative intéressante est l'extraction par microchirurgie, sous contrôle échographique, de la tumeur, ce qui permet de préserver le testicule en cas de tumeur bénigne [20];

- unique, supérieur à $1 \mathrm{~cm}$, hypoéchogène, palpable ou non, vascularisé en mode Doppler.

Ce type de nodule est toujours suspect d'un cancer et doit être enlevé systématiquement.

L'incidence du cancer testiculaire, qui touche l'homme jeune en période de fertilité, augmente dans les contrées industrialisées : un doublement de l'incidence est attendu dans les 15 à 20 années prochaines.

Parallèlement, on observe une baisse générale de la qualité du sperme. La relation entre ces deux observations n'est pas totalement élucidée, mais on postule l'existence de facteurs étiologiques communs (dysgénésie testiculaire, cryptorchidie) [21].

Le cancer testiculaire est lui-même associé à une altération de la spermatogenèse qui peut aller jusqu'à l'oligospermie sévère.

Les mécanismes invoqués sont multiples : production d'anticorps antispermatozoïdes, facteurs endocrines (testostérone basse, estradiol, bêta-HCG).

L'association entre cancer testiculaire et infertilité (avec spermogramme perturbé ou non) renforce ainsi l'idée d'un dépistage systématique $[15,22]$;

- multiple, hypoéchogène, de petite taille : dans la majorité des cas, il s'agit de nodules d'hyperplasie des cellules de Leydig, aspect échographique décrit notamment en cas de syndrome adrénogénital, mais qui peut aussi s'observer en cas de syndrome de Klinefelter ;

- unique, hyperéchogène, de petite taille : ce type de remaniement s'observe classiquement après biopsie testiculaire et correspond à un foyer cicatriciel. Il provoque généralement une absorption du faisceau d'ultrasons et peut s'accompagner d'une cheminée acoustique postérieure.

\section{Anomalie de l'échostructure testiculaire}

L'échostructure testiculaire est normalement parfaitement homogène. Occasionnellement, on observe la présence de spots hyperéchogènes intratesticulaires, soit minuscules, uniques ou multiples, groupés ou disséminés dans tout le testicule, soit plus volumineux, généralement uniques, donnant un cône d'ombre postérieur. L'entité échographique, connue sous le terme de microcalcifications intratesticulaires (MT), et décrite initialement par Backus et al. en 1994, correspond à un semis d'innombrables microformations (> 20/plan de coupe) hyperéchogènes, disséminées dans tout le testicule, donnant une échostructure hétérogène, scintillante [23]. L'histopathogénie de ces microcalcifications, nécrose cellulaire, puis formation du dépôt entouré de glycoprotéines dans un tube atrophié [24], la répartition aléatoire dans la population, avec une incidence variable suivant les études (de 0,6 à 5,6\% dans la population normale [25-27], de 0,8 à $9,5 \%$ dans la population infertile $[26,28]$ ), l'association décrite avec une multitude de situations cliniques, dont l'infertilité, la cryptorchidie [28,29], le cancer, suggèrent qu'il s'agit d'un phénomène testiculaire cellulaire au stade embryologique. Certains auteurs pensent à un phénomène commun, « toxique » pour les cellules tubulaires 
(infectieux ?, nosocomial ?, cryptorchidie), survenant au cours du développement testiculaire, et aboutissant à un syndrome de dysgénésie testiculaire qui se traduit par la nécrose cellulaire, l'atrophie tubulaire, la formation de microcalcifications et la présence de CIS [26,30-32].

L'association entre l'entité princeps MT et cancer, évoquée par Bach et al., est une idée intéressante, renforcée par cette notion de dysgénésie comme facteur précurseur commun aux microcalcifications et au CIS [33].

Cependant, elle ne fait pas l'unanimité : pour Peterson et al., cette entité est de constatation commune dans la population générale $(5 \%)$ et, ne semble pas avoir de rapport direct avec le cancer testiculaire (5/100000) [25].

Pourtant, plusieurs cas de développement de cancer sur un terrain préexistant de MT ont été publiés dans la littérature, ce qui serait plutôt en faveur de cette hypothèse.

En pratique, chez les patients azoospermiques ou oligospermiques sévères, la découverte échographique de MT bilatérale impose une surveillance échographique étroite ou une biopsie systématique des testicules à la recherche de CIS [32]. À côté de cette entité MT particulière, il existe des formes de dissémination qui diffèrent par le nombre ou par la disposition des spots intratesticulaires : la dissémination peut être globale, mais limitée $(<10$ /plan de coupe ou entre 10 et 20/plan de coupe) ou plus localisée.

L'amas localisé de microcalcifications est plus fréquemment observé en présence d'une tumeur maligne germinale ou d'un CIS [15], alors que les formes isolées de microcalcifications, extrêmement fréquentes, n'ont pas de signification pathologique démontrée. Il n'en va pas de même de la macrocalcification hyperéchogène donnant un cône d'ombre postérieur : cette anomalie est considérée comme la trace d'une tumeur éteinte (burned-out tumeur) et doit donc faire l'objet d'une description attentive.

\section{Anomalie de la vascularisation testiculaire}

La perfusion testiculaire peut être évaluée par l'échographieDoppler couleur et par l'analyse spectrale du signal artériel intratesticulaire. Le calcul de l'index de résistance (IR) est un indicateur indirect de la perfusion. L'IR est normalement compris entre 0,45 et 0,67 (branches artérielles proches du rete testis) [34].

Une élévation de l'IR intratesticulaire indique une hypoperfusion testiculaire.

Pour plusieurs auteurs, cette élévation de l'IR, chez le patient hypofertile, serait un bon indicateur d'une perturbation de la spermatogenèse $[35,36]$ et serait corrélée avec l'élévation de la FSH et la diminution du volume testiculaire en cas de varicocèle [37]. En pratique, cette mesure de l'IR intratesticulaire reste difficile (variabilité des mesures) et a peu d'influence sur la prise en charge du patient.
L'évaluation de la perfusion testiculaire a récemment bénéficié du développement de l'échographie de contraste : l'injection intraveineuse de microbulles de gaz stabilisées augmente fortement la réflectivité du sang circulant. Grâce à des algorithmes de traitement du signal généré par les bulles, il est possible d'obtenir une cartographie très précise de la perfusion testiculaire. On peut ainsi observer une très nette différence de signal entre un testicule normal et un testicule atrophié et hypoperfusé [34].

Grâce à l'utilisation de courbes de rehaussement, il est aussi possible d'obtenir une étude semi-quantitative de la perfusion.

Ce nouveau type d'investigation est potentiellement intéressant pour le choix du site de prélèvement des spermatozoïdes par extraction : comme l'ont montré Har-Toov et al., en utilisant une cartographie 3D Doppler couleur de la vascularisation intratesticulaire, on potentialise le geste en prélevant les spermatozoïdes dans les secteurs les mieux vascularisés du testicule [38]. Or, l'échographie de contraste montre beaucoup mieux la vascularisation intratesticulaire que le Doppler couleur : à l'avenir, ce nouveau mode d'investigation pourrait ainsi être utilisé pour déterminer avec précision les meilleures zones de prélèvement.

La varicocèle ou dilatation des veines du plexus pampiniforme perturbe la spermatogenèse (congestion et élévation locale de température) et aboutit à l'atrophie testiculaire : l'atrophie est proportionnelle au grade de la varicocèle et est réversible, chez l'adolescent, si le traitement est précoce $[39,40]$.

Les veines dilatées péritesticulaires sont visibles en échographie, mais la varicocèle n'est confirmée qu'en présence d'un reflux significatif enregistré en mode Doppler (> 2 secondes) [41]. L'échographie est très sensible pour détecter les formes infracliniques de varicocèle, mais la relation entre varicocèle infraclinique et infertilité est controversée $[42,43]$

\section{Anomalie morphologique du tube excréteur}

L'échographie de l'épididyme et du canal déférent (par voie externe) identifie des anomalies de calibre qui font évoquer l'existence d'un trouble excrétoire à l'origine de l'infertilité. Normalement, la tête épididymaire mesure 7,6 $\pm 1,6 \mathrm{~mm}$, le corps épididymaire 3,2 $\pm 0,8 \mathrm{~mm}$, l'anse épididymodéférentielle 7,7 $\pm 1,3 \mathrm{~mm}$, le canal déférent 1,9 $\pm 0,2 \mathrm{~mm}$ [8]. La dilatation épididymodéférentielle augmente les valeurs de mesure, mais, surtout, modifie l'aspect échographique caractéristique de l'épididyme : aspect aréolaire, voire microkystique, dépôts échogènes dans les tubules dilatés [44].

Le canal déférent dilaté est aisément repéré dans le cordon spermatique avant son entrée dans la cavité abdominale grâce à un aspect en cocarde caractéristique. En présence 
d'un tel aspect, l'hypothèse d'une infertilité d'origine excrétoire sera envisagée, et la cause de la dilatation recherchée. Le plus souvent, on retrouvera une sténose du canal excréteur secondaire à un épisode infectieux antérieur. Cette sténose, souvent difficile à localiser avec précision, sera occasionnellement identifiée le long du trajet du déférent dans le cordon.

Plus rarement, la découverte d'une dilatation de l'épididyme et du déférent sera le révélateur d'une anomalie congénitale complexe du carrefour urogénital.

Dans ces cas, l'échographie du carrefour urogénital sera complétée par une résonance magnétique du carrefour urogénital, une échographie des reins et une éventuelle déférentographie. L'agénésie bilatérale des canaux déférents (CBAVD, congenital bilateral absence of the vas deferens), autre cause possible d'azoospermie d'origine excrétoire, représente $10 \%$ des causes d'hypofertilité.

Elle présente un aspect échographique typique : une tête épididymaire dilatée, très hypoéchogène, et un corps épididymaire atrophique qui s'interrompt brutalement avec absence de queue épididymaire, d'anse épididymodéférentielle et de déférent dans le cordon [45]. C'est l'absence de jonction épididymodéférentielle et de déférent qui fera suspecter l'anomalie. Les ampoules déférentielles sont toujours absentes, et les vésicules séminales sont anormales dans $90 \%$ des cas (absentes des deux ou d'un seul côté, anomalie de volume ou d'échostructure). Cette entité particulière est observée chez $98 \%$ des patients atteints de la mucoviscidose, maladie autosomique récessive, due à une mutation du gène codant pour la protéine CFTR (cystic fibrosis transmembrane conductance regulator).

L'absence de canal déférent n'est pas due à une agénésie des tubules mésonéphrotiques pendant la vie embryonnaire, mais à un processus obstructif, fibrosant, secondaire, aboutissant à l'occlusion du canal déférent.

Le spectre des mutations est vaste (>900 décrites), et la pénétrance de l'expression du gène est variable, ce qui explique les formes incomplètes de la maladie avec conservation d'une partie de la voie séminale de l'un ou des deux côtés.

L'agénésie des déférents est rarement congénitale (défaut de développement de l'ensemble des canaux de Wolf) et s'accompagne alors d'une agénésie rénale.

\section{Conclusion}

La richesse des informations échographiques comparée à la relative pauvreté des signes cliniques rend l'échographie indispensable dans la mise au point de l'infertilité masculine. En pratique, que recherche le radiologue lors d'une échographie scrotale chez le patient hypofertile ? Dans cette population à risque, particulièrement lorsqu'il y a antécédent de cryptorchidie, l'échographie scrotale a essentiellement pour buts :

- d'évaluer la position, le volume et l'échogénicité du testicule, surtout en cas d'ectopie, d'hydrocèle ou d'anomalie à la palpation ;

- de dépister une pathologie occulte : nodule infraclinique, MT bilatérale ;

- de confirmer la présence d'une tumeur testiculaire ;

- de surveiller les patients porteurs de nodule infraclinique et/ou de MT bilatérales ;

- de dépister les signes d'obstruction canalaire ;

- de confirmer l'existence d'une varicocèle ;

- d'étudier la perfusion testiculaire afin d'orienter le geste de prélèvement de spermatozoïdes.

Cet examen doit être proposé à tout patient qui consulte pour infertilité, au même titre que le bilan biologique et spermiologique, car il oriente la mise au point, permet la détection de pathologie occulte et influence la stratégie thérapeutique.

Déclaration de conflit d'intérêt : L'auteur déclare ne pas avoir de conflit d'intérêt.

\section{Références}

1. Bruneteau L, Fauchier F, Fernandez P, et al (2000) Évaluation échographique de l'infertilité masculine. J Radiol 81:1693-700

2. Goullet E, Rigot JM, Blois N, et al (2000) Intérêt de l'échographie scrotale systématique dans la prise en charge de l'homme infertile : étude prospective de 609 cas. Progrès en urologie 10: 78-82

3. Pierik F, Dohle G, van Muiswinkel J (1999) Is routine scrotal ultrasound advantageous in infertile men? J Urol 162:1618-20

4. Onur MR, Firdolas F, Onur R, et al (2008) Scrotal ultrasonography: should it be used in routine evaluation of infertile men? Andrologia 40:58-61

5. Huyghe E, Izard V, Rigot JM, et al (2008) Les membres du Comité d'andrologie de l'Association française d'urologie (CCAFU). Prog Urol 18:95-101

6. Société française de radiologie (2009) Exploration du scrotum : échographie. Guide pratique à l'usage des médecins radiologues pour l'évaluation de leurs pratiques professionnelles, génitourinaire (URO). Fiche 4:176-9

7. Puttemans T (2005) Contenu scrotal : techniques d'exploration, indications, radioanatomie et variantes. In: Helenon $\mathrm{O}(\mathrm{ed})$ Imagerie de l'appareil génito-urinaire. Flammarion, Paris, pp. 1054-67

8. Puttemans T, Delvigne A, Murillo D (2006) Normal and variant appearances of the adult epididymis and vas deferens on highresolution sonography. J Clin Ultrasound 34:385-92

9. Sakamoto H, Ogawa Y, Yoshida H (2008) Relationship between testicular volume and testicular function: comparison of the Prader orchidometric and ultrasonographic measurements in patients with infertility. Asian J Androl 10:319-24

10. Brunereau L (2006) Imagerie de l'hypofertilité masculine. EMC (Elsevier SAS, Paris), Radiodiagnostic - Urologie-gynécologie, 34-510-A-10 
11. Bujan L, Mieusset R, Mansat A, et al (1989) Testicular size in infertile men: relationship to semen characteristics and hormonal blood levels. Br J Urol 64:632-7

12. Moon MH, Kim SH, Cho JY, et al (2006) Scrotal US for evaluation of infertile men with azoospermia. Radiology 239:168-73

13. Lenz S, Thomsen JK, Giwercman A, et al (1994) Ultrasonic texture and volume of testicles in infertile men. Hum Reprod 9: $878-81$

14. Carmignani L, Morabito A, Gadda F, et al (2005) Prognostic parameters in adult impalpable ultrasonographic lesions in severe male infertility. J Urol 174:1035-8

15. Negri L, Benaglia R, Fiamengo B, et al (2008) Cancer risk in male factor-infertility. Placenta 29(Suppl B):178-83

16. Raman JD, Nobert CF, Golstein M (2005) Increased incidence of testicular cancer in men presenting with infertility and abnormal semen analysis. J Urol 174:1819-22

17. Eifler JB Jr, King P, Schlegel PN (2008) Incidental testicular lesions found during infertility evaluation are usually benign and may be managed conservatively. J Urol 180:261-4

18. Mancini M, Carmignani L, Gazzano G, et al (2007) Highprevalence of testicular cancer in azoospermic men without spermatogenesis. Hum Reprod 22:1042-6

19. Kliesch S, Bergmann M, Hertle L, et al (1997) Semen parameters and testicular pathology in men with testicular cancer and contralateral carcinoma in situ or bilateral testicular malignancies. Hum Reprod 12:2830-5

20. Hopps CV, Goldstein M (2002) Ultrasound guided needle lolalization and microsurgical exploration for incidental non palpable testicular tumors. J Urol 168:1084-7

21. Jacobsen R, Bostofte E, Engholm G, et al (2000) Risk of testicular cancer in men with abnormal semen characteristics: cohort study. BMJ 321:789-92

22. Simon S, Lee R, Mulhall J (2001) Should all infertile males undergo urologic evaluation before assisted reproductive technologies? Two cases of testicular cancer presenting with infertility. Fertil Steril 75:1226-7

23. Backus M, Mack L, Middleton W, et al (1994) Testicular microlithiasis: imaging appearances and pathologic correlation. Radiology 192:781-5

24. Renshaw A (1998) Testicular calcifications: incidence, histology and proposed pathological criteria for testicular microlithiasis. J Urol 160:1625-8

25. Peterson AC, Bauman JM, Light DE, et al (2001) The prevalence of testicular microlithiasis in an asymptomatic population of men 18 to 35 years old. J Urol 166:2061-4

26. von Eckardstein S, Tsakmakidis G, Kamischke A, et al (2001) Sonographic testicular microlithiasis as an indicator of premalignant conditions in normal and infertile men. J Androl 22: 818-24

27. Bach AM, Hann LE, Shi W, et al (2003) Is there an increased incidence of contralateral testicular cancer in patients with intratesticular microlithiasis? J Urol 170:1040-1
28. Thomas K, Wood S, Thompson A, et al (2000) The incidence and significance of testicular microlithiasis in a subfertile population. Br J Radiol 73:494

29. Nicolas F, Dubois R, Laboure S, et al (2001) Testicular microlithiasis and cryptorchism: ultrasound analysis after orchdopexy. Prog Urol 11:357

30. Skakkebaek NE, Holm M, Hoei-Hansen C, et al (2003) Association between testicular dysgenesis syndrome (TDS) and testicular neoplasia: evidence from 20 adults patients with signs of mal development of the testis. APMIS 111:1-9

31. Holm M, Hoei-Hansen CE, Rajpert-De meyts E, et al (2003) Increased risk of carcinoma in situ in patients with testicular germ cell cancer with ultrasonic microlithiasis in the contralateral testicle. J Urol 170:1163-7

32. De Gouveia Brazao C, Pierik F, Oosterhuis J, et al (2004) Bilateral testicular microlithiasis predicts the presence of the precursor of testicular germ cell tumors in subfertile men. J Urol 171:158-60

33. Bach AM, Hann LE, Hadar O, et al (2001) Testicular microlithiasis: what is its association with testicular cancer? Radiology 220:70-5

34. Schurich M, Aigner F, Frauscher F, Pallwein L (2009) The role of ultrasound in assessment of male infertility. Eur J Obstet Gynecol Reprod Biol 144(Suppl 1):S192-S8

35. Pingerra GM, Mitterberger M, Bartsch G, et al (2008) Assessment of the intratesticular resistive index by color doppler ultrasonography measurements as a predictor of spermatogenesis. BJU Int 10(2):722-6

36. Herwig R, Tosun K, Schuster A, et al (2007) Tissue perfusion controlled guided biopsies are essential for the outcome of testicular sperm extraction. Fertil Steril 87(5):1071-6

37. Battaglia C, Pasini A, Mancini F, et al (2005) Role of intratesticular ultrasonographic and doppler flow analyses in evaluating gonadal status in male survivors of childhood malignancy. Fertil Steril 83:1867-70

38. Har-Toov J, Eytan O, Hauser R, et al (2004) A new power Doppler ultrasound guiding technique for improved testicular sperm extraction. Fertil Steril 81:430-4

39. Zini A, Buckspan M, Berardinucci D, et al (1998) Loss of left testicular volume in men with clinical left varicocele: correlation with grade of varicocele. Arch Androl 41:37-41

40. Sayfan J, Siplovich L, Koltun L, et al (1997) Varicocele treatment in pubertal boys prevents testicular growth arrest. J Urol 157:1456-7

41. Cornud F, Belin X, Amar E, et al (1999) Varicocele: strategies in diagnosis and treatment. Eur Radiol 9:536-45

42. Silber SJ (2001) The varicocele dilemma. Hum Reprod Update 7: 70-7

43. Gat Y, Bachar GN, Zukerman Z, et al (2004) Varicocele: a bilateral disease. Fertil Steril 81:424-9

44. Puttemans T (2002) Pathologie non tumorale des bourses. Encycl Med Chir (Elsevier SAS. Paris), Radiodiagnostic - Urologiegynécologie, 34-450-A-20, 15p

45. Cornud F, Belin X, Amar E, et al (1997) Imaging of obstructive azoospermia. Eur Radiol 7:1079-85 